\title{
Factores relacionados al rendimiento académico en estudiantes del máster en Salud Sexual y Reproductiva, UNAN-Managua
}

\author{
Factors related to the academic performance in master student in \\ Sexual and Reproductive Health, UNAN-Managua
}

Ximena Gutiérrez Gómez ${ }^{1}$

\section{RESUMEN}

Este ensayo explora el rendimiento académico, la evolución de sus definiciones por diferentes autores y los factores relacionados en estudiantes universitarios. Los factores relacionados, se encuentran planteados desde diversas disciplinas particularmente la psicología y la pedagogía. Con base al análisis de la información revisada, se concluye que el rendimiento académico es multifactorial, un indicador del desarrollo humano y un reflejo de las inequidades sociales. Como parte de la revisión se propone un Modelo Ecológico del Rendimiento Académico en estudiantes universitarios de la Maestría en Salud Sexual y Reproductiva, desde cuatro dimensiones: personal, familiar, institucional y social, que incluye en cada una de ellos aspectos que no fueron abordados por los autores revisados, representando un aporte de la autora que debe ser consensuado para aplicarlo en un futuro en estudiantes de pos grado.

Palabras clave: factores; rendimiento académico; universidad; alumnos; estudiantes universitarios.

\begin{abstract}
This essay explores the academic performance, the evolution of its definitions by the different authors and the factors related to the university students. The related factors, are stated from diverse subjects particularly psychology and pedagogy. Based on the analysis of the revised information, it is concluded that the academic performance has multiple factors, an indicator of human development and a reflection of social inequities. As part of the revision it is proposed an ecological model of the academic performance in the in master student in Sexual and reproductive health, from four dimensions: personal, familiar, institutional and social, each aspects include aspects that were not analyzed by the revised authors. It represents an improvement from the author which must be agreed upon to apply in the future in post-graduate students.
\end{abstract}

Keywords: factors; academic performance; university; students; university students.

DOI: https://doi.org/10.5377/multiensayos.v3i6.9683

Recibido: 23 de noviembre de 2017

Aceptado: 12 de diciembre de 2017

1 Docente UNAN Managua. Facultad de Ciencias Médicas. Correo electrónico: ximenagutiérrez644@gmail.com 


\section{INTRODUCCIÓN}

El propósito del presente artículo es analizar los factores que influyen en el rendimiento académico de estudiantes universitarios, basado en la búsqueda, selección, organización y disposición de fuentes de información, a partir del análisis de los contenidos en las fuentes consultadas.

En los últimos años las Universidades se enfrentan a nuevos retos y desafíos en la formación de recursos humanos para que sean competitivos ante un mundo laboral demandante de una fuerza de trabajo con mayores conocimientos, habilidades y destrezas en su desempeño, que aporten al desarrollo socio económico del país, contribuyendo a la reducción de la pobreza y al desarrollo humano.

La Universidad Nacional Autónoma de Nicaragua, UNAN-Managua está comprometida con la formación del talento humano, que permita generar capacidades en las personas para el mejoramiento de sus vidas y el bienestar humano. Actualmente la institución se encuentra en un proceso de auto evaluación permanente, para responder a las necesidades locales, nacionales y regionales, con calidad tanto del personal que labora en esta, como de los programas y del aprendizaje.

Un tema de interés en este contexto, es el rendimiento académico del estudiantado universitario que constituye un factor imprescindible en el abordaje del tema de la calidad de la educación superior, debido a que es un indicador que permite una aproximación a la realidad educativa (María, 2007). Probablemente es una de las dimensiones más importantes en el proceso de enseñanza aprendizaje.

El rendimiento académico de los estudiantes es un componente clave para determinar si una institución está alcanzando sus objetivos educativos. De aquí que sea esencial la existencia de un Programa de Evaluación para documentar el rendimiento académico de los estudiantes (Rodríguez Sebastián, 2004).

En estudiantes universitarios es un indicador trascendental para la evaluación de la calidad educativa, se le considera como el resultado de la unión de diferentes elementos que interactúan en el desempeño de la vida académica del estudiantado (De Miguel, Apocada, Arias, Escudero, Rodríguez y Vidal, 2002).

\section{DESARROLLO}

Conceptualizar el rendimiento académico es difícil, debido a su multicausalidad y complejidad, ya que involucra factores como: actitudes, hábitos, carácter del personal docente, metodologías, formación profesional, ambiente familiar, organización del sistema educativo y condición socioeconómica, entre otros aspectos sociales, económicos y psicológicos (Valverde, 2005). En lo que parece haber consenso es que múltiples factores afectan el rendimiento académico: personales, sociales, económicos e institucionales. (Garbanzo Vargas Guissell María, 2014). 


\section{Definición de Rendimiento Académico}

Para Carpio (1975) el rendimiento académico es el proceso técnico pedagógico que juzga los logros de acuerdo a objetivos de aprendizaje previstos. En cambio, González (1975) adopta una postura más integradora, pues lo describe como el fruto del sistema educativo, de la familia y del propio alumno, definición que caracteriza al rendimiento académico como un "producto". Por su parte, para Muñoz (1977), no se consideraron en las definiciones anteriores factores que inciden en el éxito o fracaso escolar, como son las condiciones sociales, las actitudes, el esfuerzo del estudiante, entre otros.

Los anteriores aportes dieron como resultado una evolución del concepto del rendimiento académico. Chadwick (1979) define el rendimiento académico como la expresión de capacidades y características psicológicas del estudiante desarrolladas y actualizadas a través del proceso de enseñanza aprendizaje que le posibilita obtener un nivel de funcionamiento y logros académicos a lo largo de un período o semestre, que se sintetiza en un calificativo final (cuantitativo en la mayoría de los casos) evaluador del nivel alcanzado.

Por otra parte, Caravaña (1980), es el resultado de las mediciones sociales y académicas relevantes. González P. (1982) señala que el rendimiento académico, además de las calificaciones obtenidas, debe guardar una relación entre el número de materias aprobadas y aplazadas, el tiempo que tarda el estudiante en graduarse. Jiménez R. (1983), establece que el rendimiento académico es el promedio ponderado de notas obtenidas por el alumno durante un período académico.

Adicionalmente Touron (1985), lo define como el resultado del aprendizaje, suscitado por la actividad educativa del profesor y producido en el alumno, aunque es claro que no todo aprendizaje es producto de la acción docente.

Kaczynka (1986), afirma que el rendimiento académico es el fin de todos los esfuerzos y todas las iniciativas del maestro que se juzga por los conocimientos adquiridos por los alumnos. Por su parte, en este mismo año Novaez, sostiene que el rendimiento académico es QUANTUM obtenido por el individuo en determinada actividad académica. Marcos (1987), afirma que el rendimiento académico es la utilidad o provecho de todas las actividades tanto educativas como informativas, las instructivas o simplemente nocionales.

Para Bloom (citado en Page, 1990), el rendimiento académico es lo necesario para que el estudiante sea capaz de llevar a la práctica sus conocimientos, que pueda aplicar la información adquirida a la solución de problemas; en pocas palabras, es el resultado del trabajo escolar (Patricia, 2012). Por su parte Quezada (1991), asegura que es el resultado de la evaluación del aprendizaje. Para Austin (1993), el rendimiento escolar influye en la vida personal del estudiante, tanto en el éxito como en el fracaso (escolar y persona); es parte de su historia, de su vivencia, familia, nivel socio económico, entre otros, afectando su autoestima, motivaciones e intereses. 
En igual forma, Larrosa, Faustino (1994), refiere que el rendimiento académico, es la expresión de capacidades, habilidades y destrezas cognitivas, que el estudiante desarrolla durante el proceso de enseñanza aprendizaje (citado por Luis, 2013). Alfonso S. (1994), señala que el rendimiento académico es el resultado de la acción escolar, que expresa el éxito alcanzado por el estudiante en el aprovechamiento del $100 \%$ de los objetivos contemplados en el programa de estudio de las asignaturas impartidas, detectado por la evaluación integral y condicionada por los diversos factores escolares y sociales.

Según Figueroa, Marcos (1995) señala que el rendimiento académico sintetiza la acción del proceso educativo, no solo en el aspecto cognitivo, sino en el conjunto de habilidades, destrezas, aptitudes, intereses del alumno. Para el logro de un eficiente rendimiento académico del estudiante intervienen una serie de factores educativos: metodología del profesor, el aspecto individual del alumno, apoyo familiar, entre otros (citado por Luis, 2013). Marco, A. (1996) afirma que el rendimiento académico es la utilidad o provecho que estudiante obtiene de todas las actividades educativas como informales que el alumno enfrenta durante la escuela.

Fermín, I. (1997), define rendimiento académico como el promedio de notas obtenidas en cada lapso. Aranda (1998), considera que es el resultado del aprovechamiento académico en función a diferentes objetivos y hay quienes homologan que el rendimiento académico puede ser definido como el éxito o fracaso en el estudio expresado a través de notas y calificativos. Se define como el progreso alcanzado por los alumnos en función de los objetivos programáticos previstos, es decir, según los objetivos que se han planificado, por tanto y que tan rápido avanza el alumnado dando los resultados más satisfactorios posibles.

Según (Kerlinger, 1998), la educación universitaria es un hecho intencionado y en términos de calidad educativa busca permanentemente mejorar el aprovechamiento del alumno. En este sentido la variable dependiente clásica en la educación superior es el rendimiento.

Según Miranda, Miguel (2004), señala que el eficiente rendimiento académico que obtenga el alumno, se deberá a diversos factores externos (metodología del profesor, el ambiente de clase, la relación familiar, el programa educativo, entre otros) e internos (actitud, personalidad, motivación $\mathrm{y}$ autoconcepto que tenga el alumno en clase). El rendimiento académico de los estudiantes universitarios constituye un factor imprescindible y fundamental para la valoración de la calidad educativa en la enseñanza superior (María, 2007).

De todas estas definiciones se puede reflexionar que el rendimiento académico es producto de múltiples factores: individuales, familiares, institucionales y sociales que está mediado por el proceso de enseñanza aprendizaje, él o la docente y se refleja de forma objetiva a través de calificaciones o notas. Basado en las definiciones encontradas, también se puede considerar un indicador de la calidad educativa en el que influye la estructura institucional, los procesos de enseñanza-aprendizaje y los resultados, que en este caso son: las calificaciones. 
Al analizar las definiciones de los diferentes autores se logra identificar que el rendimiento académico universitario presenta algunas variaciones a las definiciones revisadas, es complejo de medir en estudiantes de pos grado, ya que su medición amerita un enfoque holístico, integral, una filosofía educacional y forma constructivista que debe considerar los diferentes escenarios en que los/as estudiantes se han desarrollado: su historia de vida, los antecedentes previos en la educación secundaria, su capacidad intelectual, sus hábitos de estudio, el vínculo que exista entre la comunidad, el mundo natural y sus valores, como un ser único, pero que está en interacción permanente con el mundo que le rodea.

Los aspectos antes mencionados permiten identificar que las calificaciones obtenidas por un estudiante no reflejan integralmente sus logros alcanzados, quedando brechas que deben ser consideradas por las instituciones educativas. ¿Qué hacer con estudiantes que tienen dificultades familiares y un bajo rendimiento académico? Es posible considerar que las calificaciones son suficientes para valorar el rendimiento académico de estudiantes universitarios de pos grado?, ¿Qué se puede hacer para considerar los aspectos individuales como la motivación, la actitud y la personalidad como parte del éxito o fracaso del aprendizaje?¿Qué instrumentos pueden ser utilizados para considerar los otros factores que influyen en el rendimiento académico que no son propiamente los individuales?

Basado en las definiciones encontradas se debe considerar al rendimiento académico como un indicador educativo del Desarrollo Humano, (PNUD 1992:18), es un concepto amplio e integral basado en la idea de bienestar de la población, que ayuda a distinguir dos aspectos: uno, es la formación de capacidades humanas como un mejor estado de salud o mayores conocimientos; el otro, es el grado en el que los individuos emplean las capacidades adquiridas. Así mismo, se debe considerar que es el reflejo de inequidades sociales, dado que aunque algunos estudiantes tengan factores personales positivos para alcanzar un buen rendimiento académico, este puede ser afectado por las condiciones económicas de su familia, y la universidad donde estudia.

García y Palacios (1991), después de realizar un análisis comparativo de diversas definiciones del rendimiento académico concluyen que hay un doble punto de vista: estático y dinámico, caracterizándolo de la siguiente manera: a) El rendimiento en su aspecto dinámico responde al proceso de aprendizaje, como tal está ligado a la capacidad y esfuerzo del alumno, b) en su aspecto estático comprende al producto del aprendizaje generado por el alumno y expresa una conducta de aprovechamiento, c) está ligado a medidas de calidad y juicio de valoración, d) es un medio y no un fin en sí mismo, e) está relacionado a propósitos de carácter ético que incluye expectativas económicas lo cual hace necesario un tipo de rendimiento en función al modelo (Vildoso, 2003).

\section{Teorías del Rendimiento Académico}

Existen diferentes teorías que explican el rendimiento académico. Kaczynska (1963), afirma que tradicionalmente se creía que el rendimiento académico era producto de la buena o mala voluntad 
del alumno olvidando otros factores que pueden intervenir en el rendimiento académico. Quiroz (2001), hace referencia al rendimiento basado en la voluntad, atribuyendo la capacidad del hombre a su voluntad.

Otra teoría es la del rendimiento académico basado en la capacidad. Esta postura sostiene que el rendimiento académico está determinado no solo por la dinamicidad del esfuerzo, sino también por los elementos con los que el sujeto se halla dotado, ejemplo, la inteligencia.

En la revisión literaria se encuentra documentada la denominación de rendimiento académico en autores como Nieto (2008) y en su texto titulado, "Hacia una teoría sobre el rendimiento académico en enseñanza primaria a partir de la investigación empírica: datos preliminares", explicando que sobre el tema se han realizado estudios con diferentes modelos de investigación, como el exploratorio, descriptivo y explicativo y advirtiendo que a pesar de que hay información sobre el tema esta no es concluyente (Oscar, Octubre-Marzo 2012).

Cuando se trata de evaluar el rendimiento académico y como mejorarlo, se analizan en mayor o menor grado los factores que pueden influir en él, generalmente se consideran, entre otros, factores socio económicos, la amplitud de los programas de estudio, las metodologías de enseñanza utilizadas, la dificultad de emplear una enseñanza personalizada, los conceptos previos que tienen los estudiantes, así como el nivel de pensamiento formal de los mismos (Benítez, Giménez y Oscika, 2000). Sin embargo, Jiménez (2000) refiere que "se puede tener una buena capacidad intelectual y buenas aptitudes, sin embargo, no estar obteniendo un rendimiento adecuado" ante la disyuntiva y con la perspectiva que el rendimiento académico es un fenómeno multifactorial (Rubén, 2003).

Para algunos autores (De Miguel, 2001), es necesario distinguir entre rendimiento inmediato (notas) y el mediato (logros personales y profesionales. Latiesa (1992) diferencia el rendimiento en sentido amplio (éxito, retraso y abandono) y en sentido estricto (notas) o regularidad académica. Finalmente es de interés el concepto de rendimiento académico como la relación entre la potencia, es decir, el input, del que aprende y el producto que se logra, el aprendizaje (Rodríguez Sebastián, 2004).

Como se puede observar a lo largo de las diferentes investigaciones citadas, el análisis sobre el rendimiento académico muestra una gran diversidad de líneas de estudio, lo que permite no solo comprender su complejidad sino su importancia dentro del acto educativo (Rubén, 2003).

El rendimiento académico ha sido estudiado con diferentes enfoques teóricos y metodológicos, además de lo complejo que resulta la definición del rendimiento académico, la medición del mismo representa un reto para las universidades, porque convergen distintas variables y formas de evaluación que dependen del objetivo de las materias o de cada profesor. Sin embargo, los 
indicadores más utilizados para el rendimiento académico han sido las calificaciones, que por lo general son cuantitativas.

En nuestro país el rendimiento académico se mide cuantitativamente mediante las calificaciones que se encuentran entre un mínimo de 0 y un máximo de 100, a través de este método se valora el aprendizaje del estudiante y el logro de los objetivos de las asignaturas que corresponden a su carrera. En este contexto las calificaciones representan el reconocimiento social y legal del rendimiento académico en el ámbito universitario, a pesar, que solamente poseen un valor relativo y no holístico del estudiante

Sin embargo, el rendimiento académico debe medirse mediante indicadores de resultados derivados de la labor universitaria o mediante indicadores de proceso educativo (Esparrells, 2012). Los primeros se refieren a la adquisición de competencias, inserción laboral y empleabilidad, mientras que los segundos se miden con tasas estandarizadas, como la tasa de abandono, la de rendimiento y la de éxito.

Rodríguez (2004), apunta, refiriéndose a la tasa de abandono, a la diversidad de tipologías: abandono por expulsión de la institución universitaria, abandono completo de los estudios o abandono transitorio (abandono de la universidad o de la carrera inicial para trasladarse a una nueva universidad y cursar otros estudios). Sin estos matices, la tasa de abandono proporciona una información parcial que hay que contrastar con datos adicionales.

Un estudio realizado por el Instituto de Educación Superior en México señala como indicadores del rendimiento académico la deserción, el rezago estudiantil y los bajos índices de eficiencia terminal, que se encuentran entre los problemas más complejos y frecuentes de las instituciones de la educación superior.

En el ámbito de las universidades, el término deserción se define como el abandono de los cursos o la carrera en que se ha inscrito el estudiante. Según la teoría del abandono propuesta por Tinto (1987), existen modalidades: abandono definitivo del sistema educativo, por transferencia a otra institución y cambio de carrera después de haber estado un tiempo en ellas.

Camarena (1985), señala que la eficiencia terminal en la educación superior se conceptualiza como la relación entre el número de estudiantes que se inscriben por primera vez en una carrera profesional y los que logran finalizar sus carrera de la misma generación, después de acreditar todas las materias correspondientes al plan de estudios en los tiempos estipulados.

Villa J. y Pacheco (2002), plantean que históricamente, la eficiencia terminal ha sido mejor en las instituciones privadas que en las públicas. Sin embargo, el hecho de alcanzar los niveles de escolaridad más altos no garantiza que los estudiantes sean absorbidos por el mundo laboral. 
En el país no existen antecedentes o si existen no están disponibles de estudios relacionados con el rendimiento académico, sus indicadores y factores, siendo estos elementos medulares para conocer la calidad educativa en la educación superior.

\section{Factores que influyen en el Rendimiento Académico de los estudiantes universitarios}

Según (Luis, 2013) existen dos factores: a) factores intrínsecos: dentro de estos factores se destacan: la inteligencia, memoria, atención, motivación, concentración, predisposición al estudio, la actitud frente al docente, las emociones de alegría, tristeza, entre otros. Así por ejemplo, si un alumno presenta un coeficiente intelectual alto, generara un buen rendimiento académico, siempre y cuando las emociones, actitudes y motivaciones no influyen en él, b) factores extrínsecos: dentro de estos factores se encuentran: el aspecto familiar y social.

Adicionalmente, un estudio realizado por Quiroz (2001) citado por (Quiñonez, 1998), menciona que existen dos condicionantes del rendimiento académico: a) Factores endógenos: relacionados directamente a la naturaleza psicológica o somática del alumno manifestándose estas en el esfuerzo personal, motivación, predisposición, nivel de inteligencia, hábitos de estudio, actitudes, ajuste emocional, adaptación al grupo, edad cronológica, estado nutricional, deficiencia sensorial, perturbaciones funcionales y el estado de salud física entre otros, b) Factores exógenos: son los factores que influyen desde el exterior en el rendimiento académico. En el ambiente social se menciona el nivel socioeconómico, procedencia urbana o rural, conformación del hogar, entre otros. En el ámbito educativo se incluyen la metodología del docente, los materiales educativos, material bibliográfico, infraestructura, sistemas de evaluación, entre otros.

Anterior a Quiroz y Luis, Mitchell, Hall y Pratkowska (1975), realizaron una investigación donde destacan siete factores en el rendimiento académico: 1) Ambiente de estudio inadecuado; 2) Falta de compromiso con el curso (motivación e interés por las materias que componen el plan de estudios); 3) Objetivos académicos y vocacionales no definidos; 4) Ausencia de análisis de la conducta del estudio (tiempo que se invierte en el estudio personal, asistencia a clases y establecimiento de prioridades para llevar a cabo las demandas académicas; 5) Presentación con ansiedad a los exámenes escritos; 6) Presentación de ansiedad académica (está asociado con la ejecución en seminarios, dirección de grupos pequeños o grandes, exposiciones de temas); 7) Deficiencia en hábitos de habilidades de estudio (frecuencia del empleo de estas habilidades.

Por su parte Goleman (1996), citado por (Quiñonez, 1998), relaciona el rendimiento académico con la inteligencia emocional, señalando que los objetivos a alcanzar son los siguientes: confianza, curiosidad, intencionalidad, autocontrol, relación, capacidad de comunicar y cooperación.

Por otro lado, (Vásquez Claudia M, 2012), hace referencia a algunos factores condicionantes del rendimiento académico universitario, en la que se abandonan los enfoques reduccionistas que intentan analizar el rendimiento académico desde una variable única, a una enfocada en múltiples 
factores interrelacionados. A nivel mundial algunos autores se han enfocado en la búsqueda y análisis de los factores preponderantes. Se destaca también el estudio de Garbanzo Vargas (2007) que sostiene la existencia de diferentes aspectos asociados al rendimiento académico, tanto internos como externos al individuo y los agrupa en factores de orden social, cognitivo y emocional, y los clasifica en tres determinantes: sociales, personales e institucionales.

Según un estudio realizado por Vázquez Claudia M., Cavallo Marcela A, Aparicio Silvia N. factores de impacto en el rendimiento académico universitario. Un estudio a partir de las percepciones de los estudiantes, publicado en Noviembre 2012, refuerza los factores relacionados con el rendimiento académico, destacando los factores personales, sociales e institucionales.

En este mismo documento se destacan entre los factores personales: competencia cognitiva, motivación, condiciones cognitivas, auto concepto académico, autoeficacia percibida, bienestar psicológico, satisfacción y abandono con respecto a los estudios, asistencia a clase, aptitudes, sexo, formación académica previa, nota de acceso a la universidad.

Entre los factores sociales se hace mención a diferencias sociales, entorno familiar, nivel educativo de los progenitores, nivel educativo de la madre, contexto socio económico, variables demográficas.

Los factores institucionales mencionados son: elección de los estudios según interés del estudiante, complejidad en los estudios, condiciones institucionales, servicios institucionales de apoyo, ambiente estudiantil, relación estudiante-profesor, pruebas específicas de ingreso a la carrera.

En sus estudios con García-Valcárcel Muñoz-Repiso, A (2007), Tejedor Tejedor identifica cinco tipo de variables explicativos del rendimiento: Variables de identificación (género, edad), Variables psicológicas (aptitudes intelectuales, personalidad, motivación, estrategias de aprendizaje, entre otros, Variables académicas (tipos de estudios cursados, curso, opción en que se estudia una carrera, rendimiento previo, entre otros.), Variables pedagógicas (definición de competencias de aprendizaje, metodología de enseñanza, estrategias de evaluación, entre otros.) y Variables sociofamiliares (estudios de los padres, profesión, nivel de ingresos, entre otros.) .

En el estudio de Valle Arias et al, resalta la clasificación en variables dependientes (endógenas) y variables independientes (exógenas). Las endógenas se refieren a las atribuciones causales, Autoconcepto académico, enfoques de aprendizaje, rendimiento académico. Las exógenas, al rendimiento previo, concepción incremental de la inteligencia, capacidad percibida, percepción de criterios de evaluación, análisis de las características de la tarea, percepción del estilo de enseñanza y percepción del tipo de materia.

Por otro lado (Avalos Abel, 2014) refiere que existen modelos causales del rendimiento académico. En este se centra (Tejedor i García-Varcárcel, 2006) en aspectos motivacionales. Otros, ponen énfasis en el papel de las instituciones y de sus políticas (Michavilla i Esteve 2011) y otros 
focalizan en los desajustes entre las expectativas que se crea el alumnado sobre la universidad y las oportunidades y la realidad que brinda ya que a menudo estas expectativas creadas son demasiado elevadas y defraudan a los alumnos de nuevo ingreso (Masjuan, Elías i Troiano 2009).

En el ámbito universitario se han realizado diversos estudios enfocados en explicar el rendimiento de los alumnos (Patricia, 2012) y si bien las variables explicativas varían de acuerdo con el nivel educativo, la mayoría considera el nivel de estudios de los padres, personalidad, inteligencia, hábitos de estudio y rendimiento anterior, entre otras (Herrera, Nieto, Rodríguez y Sánchez, 1999).

Después de las consideraciones anteriores es evidente que existen múltiples factores relacionadas con el rendimiento académico, los que han sido abordados desde diferentes perspectivas, por lo que se puede concluir, que el rendimiento académico es dinámico y los procesos que influyen en el desarrollo son continuos e interactivos y responden a un sistema ecológico propuesto por Bronfenbrenner (1979), que reconoce la influencia que tienen los ambientes en el desarrollo de la persona.

El Modelo Ecológico propuesto por Bronfenbrenner plantea su visión ecológica del desarrollo humano, destacando la importancia crucial al estudio de los ambientes en los que la persona recibe el ambiente que le rodea (su ambiente ecológico) y el modo en que se relaciona con él (Alberto, 2001).

Apoyados en los aspectos teóricos revisados, se puede construir una nueva teoría del rendimiento académico en estudiantes universitarios de pos grado, basado en un Modelo Ecológico, en el que se interrelacionen cuatro dimensiones: la personal, familiar, institucional y social.

La dimensión personal comprende factores referidos por diferentes autores: sexo (Antoni 2003), edad cronológica (Quiroz 2001; García Valcárcel Muñoz-Repiso, A. 2007), tiempo que se invierte en los estudios (Luis, Mitchell, Hall y Pratwoska, 1975), procedencia (Quiroz 2001), confianza, curiosidad, autocontrol, relación, capacidad de comunicar y cooperación (Goleman 1996), hábitos de estudio (Luis, Mitchell, Hall y Pratwoska, 1975), motivación (Vásquez Claudia M. 2012), calificaciones previas.

En esta dimensión además de los aportes realizados por los diferentes autores, un aporte de la autora de este ensayo a los factores que afectan a estudiantes universitarios de pos grado son los relacionados con la situación laboral, carga laboral, las adicciones como el consumo de alcohol, drogas, las tecnologías de información y comunicación, que limitan el desarrollo creativo de los/as estudiantes, así como la salud física y los estilos de vida relacionados con la sexualidad.

La dimensión familiar que incluye el entorno familiar (Vásquez Claudia M, 2012) considerando la comunicación familiar relacionada con las relaciones familiares, incluyendo la relación con la pareja, la conformación del hogar (número de hijos, familia nuclear, familia compuesta), el apoyo 
familiar que en el caso de estudiantes de pos grado muchas veces es brindado por la pareja, no precisamente por los progenitores. La situación de salud de la familia, dado que en muchos casos, tener una persona enferma representa una carga emocional que afecta el rendimiento académico de estudiantes, además de la situación económica de la familia, que en ocasiones determina el abandono de los estudios y la eficiencia terminal.

Adicionalmente en el Modelo Ecológico del rendimiento académico universitario de pos grado se destaca la dimensión institucional (relacionadas con la infraestructura, procesos y resultados), el apoyo de pares, la relación estudiante profesor, la metodología docente, el material bibliográfico disponible, los sistemas de evaluación, el número de asignaturas, la extensión de los programas, la dificultad de las materias, horario de clase, número de exámenes y trabajos.

Dado el enfoque holístico e integrador del Modelo Ecológico del rendimiento académico universitario de estudiantes de pos grado, se debe considerar la dimensión social: Políticas públicas sociales: salud, educación, el contexto socio económico, el mercado de trabajo y las oportunidades laborales de los estudiantes egresados en las diferentes carreras universitarias, la cultura, valores y pobreza.

\section{Modelo Ecológico del rendimiento académico universitario en estudiantes de pos grado}

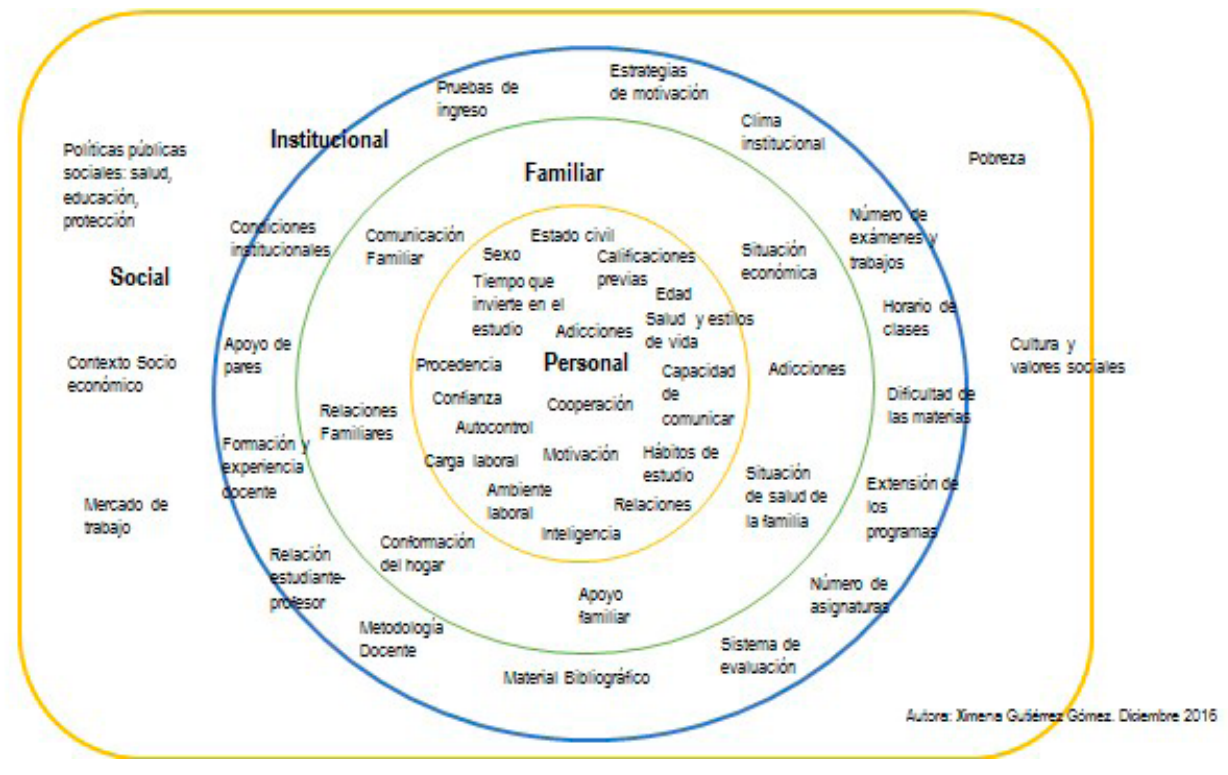

\section{CONCLUSIONES}

El rendimiento académico se aborda desde múltiples factores interrelacionados, es un indicador que refleja la calidad educativa y el desarrollo humano de un país, así como una manifestación de las inequidades sociales, por lo que su medición representa un reto para las instituciones de educación superior. 
La definición del rendimiento académico ha venido evolucionando a lo largo de los años, encontrando puntos en común y algunos vacíos desde los diferentes abordajes, que dejan por un lado los aspectos propios del estudiante universitario que no dedica tiempo completo a estudiar, tales como la carga laboral y el ambiente laboral. Así mismo, quedan a un lado, los factores relacionados con la salud, los estilos de vida de los estudiantes, la sexualidad y las adicciones; que desde la perspectiva de la autora deben ser incorporados ya que en la historia estudiantil universitaria de pos grado tienen gran importancia.

Las Universidades deben cambiar sus paradigmas y considerar la evaluación holística de los logros obtenidos por los estudiantes que considere los aspectos más relevantes de las dimensiones descritas en el Modelo Ecológico, en estudiantes de posgrado que garantice la formación de personas creativas, con habilidades sociales, capaces de adaptarse a los cambios, con valores éticos, de justicia y solidaridad.

\section{REFERENCIAS BIBLIOGRÁFICAS}

Alberto, G. S. (2001). Modelo Ecològico/Modelo Integral de intervenciòn temprana. Madrid, España: Real Patronato sobre Discapacidad.

Avalos Abel, C. B. (2014). Grupo de Trabajo de Rendimiento Académico. España: Universidad de Barcelona.

Garbanzo Vargas Guissell María. (2014). Factores asociados al rendimiento académico tomando en cuenta el nivel socio económico: Estudio de regresion múltiple en estudiantes universitarios. Revista Electrónica Educare (Educare Electronic Journal), 119-154.

Gonzalez Barbera Coral, N. C. (s.f.). Rendimiento Académico y factores asociados. Aportaciones de algunas evaluaciones a gran escala.

Luis, A. E. (2013). Autoestima y rendimiento académico de los estudiantes del X ciclo 2012- II de la Escuela Académica Profesional de Educación Primaria y problemas de aprendizaje de la Universidad Nacional José Faustino Sánchez Carrión-Huacho. Huacho, Huacho, Perú.

María, G. V. (2007). Factores asociados al rendimiento académico en estudiantes universitarios, una reflexión desde la calidad de la educación superior pública. Revista Educación 31(1), 43-63, ISSN: 0379-7082, 2007, pp. 43-63.

Montero Rojas, E. V. (22 de Octubre de 2007). Factores institucionales, pedagógicos, psicosociales y sociodemográficos asociados al rendimiento académico en la Universidad de Costa Rica: un análisis multinivel. Obtenido de http:/www.uv.es/RELIEVE : http://www.uv.es/relieve/ v13n2/RELIEVEv13n2_5.htm

Octavio, S. L. (2015). Rendimiento académico de los estudiantes de secundaria obligatoria y su relación con las aptitudes mentales y las actitudes ante el estudio. Madrid, Madrid, España.

Oscar, E. (Octubre-Marzo 2012). El rendimiento académico, un fenómeno de múltiples relaciones y complejidades. Revista Vanguardia Psicológica, 144-173.

Patricia, G. B. (2012). Modelos predictivos y explicativos del rendimiento académico universitario: caso de una institución privada en México. Madrid, Madrid, España. 
Quiñonez, A. V. (1998). Influencia de los hábitos de estudio en el rendimiento académico de los estudiantes del I año del ISTH - Huaral. Huando, Huando, Perú.

Ramón, D. S. (Mayo-ago. 2000). La calidad en el desarrollo profesional: avances y desafíos. Educación Médica Superior v.14 n.2 Ciudad de la Habana .

Rodríguez Sebastián, F. E. (2004). El rendimiento académico en la transición secundariauniversidad. Revista de Educación, núm. 334 , pp. 391-414.

Rubén, E. N. (2003). El rendimiento académico: concepto, investigación y desarrollo. Revista Electrónica Iberoamericana sobre Calidad, Eficacia y Cambio en Educación.

Vásquez Claudia M, C. M. (2012). Factores de impacto en el rendimiento académico universitario: Un estudio a partir de las percepciones de los estudiantes. El Rosario, El Rosario, Argentina. 